\title{
Minimum energy control of descriptor discrete-time linear systems by the use of Weierstrass-Kronecker decomposition
}

\author{
TADEUSZ KACZOREK and KAMIL BORAWSKI
}

\begin{abstract}
The minimum energy control problem for the descriptor discrete-time linear systems by the use of Weierstrass-Kronecker decomposition is formulated and solved. Necessary and sufficient conditions for the reachability of descriptor discrete-time linear systems are given. A procedure for computation of optimal input and a minimal value of the performance index is proposed and illustrated by a numerical example.
\end{abstract}

Key words: descriptor, discrete-time, linear, system, Weierstrass-Kronecker decomposition, minimum energy control.

\section{Introduction}

A dynamical system is called positive if its trajectory starting from any nonnegative initial condition state remains forever in the positive orthant for all nonnegative inputs. An overview of state of the art in positive system theory is given in the monographs $[8,17]$ and in the papers $[11,18-22]$. Models having positive behavior can be found in engineering, economics, social sciences, biology and medicine, etc.

Descriptor (singular) linear systems were considered in many papers and books [1-7, 9, 19, 28-30]. The positive standard and descriptor systems and their stability have been analyzed in $[17,21]$. Descriptor positive discrete-time and continuous-time nonlinear systems have been analyzed in [11].

The minimum energy control problem for standard linear systems has been formulated and solved by J. Klamka [24-26] and for 2D linear systems with variable coefficients in [24]. The relative controllability and minimum energy control problem of linear systems with distributed delays in control has been investigated by Klamka in [27]. The minimum energy control of fractional positive continuous-time linear systems has been

The Author is with Bialystok University of Technology, Faculty of Electrical Engineering, Wiejska 45D, 15-351 Bialystok, e-mail: kaczorek@isep.pw.edu.pl

This work was supported by National Science Centre in Poland under work No. 2014/13/B/ST7/03467.

Received 21.01.2016. 
addressed in [14] and for positive discrete-time linear systems in [10, 13, 15]. The minimum energy control problem for positive electrical circuits has been investigated in [16].

In this paper the minimum energy control problem for descriptor discrete-time linear systems by the use of Weierstrass-Kronecker decomposition will be formulated and solved. The paper is organized as follows. In section 2 the Weierstrass-Kronecker decomposition theorem is recalled and the necessary and sufficient conditions for the reachability of the descriptor discrete-time linear systems are given. In section 3 the minimum energy control problem of the descriptor discrete-time linear systems by the use of Weierstrass-Kronecker decomposition is formulated and solved. The procedure of finding of the optimal input sequences is proposed and illustrated by numerical example in section 4. Concluding remarks are given in section 5.

The following notation will be used: $\mathfrak{R}$ - the set of real numbers, $\mathfrak{R}^{n \times m}$ - the set of $n \times m$ real matrices, $\Re_{+}^{n \times m}$ - the set of $n \times m$ matrices with nonnegative entries and $\mathfrak{R}_{+}^{n}=\mathfrak{R}_{+}^{n \times 1}, I_{n}-$ the $n \times n$ identity matrix, $Z_{+}-$the set of nonnegative integers.

\section{Preliminaries}

Consider the descriptor discrete-time linear system

$$
E x_{i+1}=A x_{i}+B u_{i}, \quad i \in Z_{+}=\{0,1, \ldots\},
$$

where $x_{i} \in \mathfrak{R}^{n}, u_{i} \in \mathfrak{R}^{m}$ are the state and input vectors and $A \in \mathfrak{R}^{n \times n}, B \in \mathfrak{R}^{n \times m}$. It is assumed that $\operatorname{det} E=0$ and

$$
\operatorname{det}[E z-A] \neq 0 \text { for some } z \in C \text { (the field of complex numbers). }
$$

It is well-known $[12,23]$ that if (2) holds then there exist nonsingular matrices $P, Q \in$ $\Re^{n \times n}$ such that

$$
P[E z-A] Q=\left[\begin{array}{cc}
I_{n_{1}} z-A_{1} & 0 \\
0 & N z-I_{n_{2}}
\end{array}\right], A_{1} \in \stackrel{n_{1} \times n_{1}}{\operatorname{Re}}, N \in \stackrel{n_{2} \times n_{2}}{\operatorname{Re}},
$$

where $n_{1}=\operatorname{det}\{\operatorname{det}[E z-A]\}, n_{2}=n-n_{1}$ and $N$ is the nilpotent matrix with the index $\mu$, i.e. $N^{\mu-1} \neq 0, N^{\mu}=0$.

The matrices $P$ and $Q$ can be computed using procedures given in [12, 23, 29]. Premultiplying (1) by the matrix $P$ and introducing the new state vector

$$
\bar{x}_{i}=\left[\begin{array}{c}
\bar{x}_{1, i} \\
\bar{x}_{2, i}
\end{array}\right]=Q^{-1} x_{i}, \bar{x}_{1, i} \in \mathfrak{R}^{n_{1}}, \bar{x}_{2, i} \in \mathfrak{R}^{n_{2}}
$$

and using (4) we obtain

$$
P E Q Q^{-1} x_{i+1}=P A Q Q^{-1} x_{i}+P B u_{i}
$$


and

$$
\begin{gathered}
\bar{x}_{1, i+1}=A_{1} \bar{x}_{1, i}+B_{1} u_{i}, \\
N \bar{x}_{2, i+1}=\bar{x}_{2, i}+B_{2} u_{i}
\end{gathered}
$$

where

$$
P B=\left[\begin{array}{c}
B_{1} \\
B_{2}
\end{array}\right], B_{1} \in \Re^{n_{1} \times m}, B_{2} \in \Re^{n_{2} \times m} .
$$

Theorem 1 The solution $\bar{x}_{1, i}$ of the equation (6a) has the form

$$
\bar{x}_{1, i}=A_{1}^{i} \bar{x}_{10}+\sum_{k=1}^{i-1} A_{1}^{i-k-1} B_{1} u_{k} .
$$

Proof The proof is given in [12].

Theorem 2 The solution $\bar{x}_{2, i}$ of the equation (6b) for zero initial conditions $\bar{x}_{20}=0$ has the form

$$
\bar{x}_{2, i}=-\sum_{k=0}^{\mu-1} N^{k} B_{2} u_{i+k}
$$

Proof The proof is given in [12].

Definition 1 The descriptor discrete-time linear system (1) is called reachable in $q$ steps $(q \leqslant n)$ if for every given final state $x_{f} \in \mathfrak{R}^{n}$ there exists an input sequence $u_{0}, u_{1}, \ldots, u_{q-1}$ which steers the state of the system from zero initial condition $x_{0}=0$ to $x_{f}$.

Theorem 3 The descriptor discrete-time linear system (1) is reachable in q steps if and only if one of the equivalent conditions is satisfied

1) $\operatorname{Im}[E s-A]+\operatorname{Im} B=\mathfrak{R}^{n}$ for all $s \in C$ and $\operatorname{Im} E+\operatorname{Im} B=\mathfrak{R}^{n}$

2) $\operatorname{rank}\left[\begin{array}{llll}B_{1} & A_{1} B_{1} & \cdots & A_{1}^{n_{1}-1} B_{1}\end{array}\right]=n_{1}, n_{1} \leqslant q$,

$$
\operatorname{rank}\left[\begin{array}{llll}
B_{2} & N B_{2} & \cdots & N^{\mu-1} B_{2}
\end{array}\right]=n_{2}, n_{2} \leqslant q,
$$

where Im denotes the image and $n_{1}, n_{2}$ are defined by (3).

Proof The proof is given in [12]. 


\section{Problem formulation and its solution}

Consider the descriptor discrete-time linear system (1). If the system is reachable in $q$ steps, then usually there exists many different input sequences $u_{i} \in \mathfrak{R}^{m}, i=0,1, \ldots, q-1$ that steers the state of the system from $x_{0}=0$ to $x_{f}$. Among these input sequences we are looking for the sequence $u_{i} \in \mathfrak{R}^{m}, i=0,1, \ldots, q-1$ that minimizes the performance index

$$
I(u)=\sum_{k=0}^{q-1} u_{k}^{T} \widetilde{Q} u_{k}
$$

where $\widetilde{Q} \in \Re^{m \times m}$ is a symmetric defined matrix.

From the block-diagonal structure of matrices $P E Q$ and $P A Q$ it follows that minimum energy control problem can be applied to both subsystems (6) separately. The minimum energy control problem can be stated as follows.

Given the matrices $A_{1} \in \mathfrak{R}^{n_{1} \times n_{1}}, B_{1} \in \mathfrak{R}^{n_{1} \times m}, N \in \mathfrak{R}^{n_{2} \times n_{2}}, \widetilde{Q} \in \mathfrak{R}^{m \times m}$, of the performance matrix (11) and $x_{f} \in \Re^{n}$, find an input sequence $u_{i}=\left[\begin{array}{l}u_{1, k} \\ u_{2, j}\end{array}\right] \in \Re^{(l+\mu) m}$, where $l+\mu=q, u_{1, k} \in \mathfrak{R}^{l m}, k=0,1, \ldots, l-1$ and $u_{2, j} \in \mathfrak{R}^{\mu m}, j=0,1, \ldots, \mu-1$ that steers the state vector from $x_{0}=0$ to $x_{f}$ and minimizes the performance index (11).

Let us consider the subsystem (6a). To solve the problem we define the matrix

$$
W_{l}=R_{l} \widetilde{Q}_{1}^{-1} R_{l}^{T} \in \Re^{n_{1} \times n_{1}},
$$

where $R_{l}$ is the reachability matrix defined by

$$
R_{l}=\left[\begin{array}{llll}
B_{1} & A_{1} B_{1} & \cdots & A_{1}^{n_{1}-1} B_{1}
\end{array}\right]
$$

and

$$
\widetilde{Q}_{1}=\operatorname{blockdiag}\left[\begin{array}{lll}
\widetilde{Q}_{1}^{-1} & \ldots & \widetilde{Q}_{1}^{-1}
\end{array}\right] \in \Re^{l m \times l m} .
$$

If the system (1) is reachable in $q$ steps then the input sequence

$$
u_{l}=\left[\begin{array}{c}
u_{l-1} \\
u_{l-2} \\
\vdots \\
u_{0}
\end{array}\right]=\widetilde{Q}_{1}^{-1} R_{l}^{T} W_{l}^{-1} \bar{x}_{1, f} \in \Re^{l m}
$$

steers the subsystem (6a) from $\bar{x}_{10}=0$ to $\bar{x}_{1, f}$ since

$$
\bar{x}_{1, l}=R_{l} u_{l}=R_{l} \widetilde{Q}_{1}^{-1} R_{l}^{T} W_{l}^{-1} \bar{x}_{1, f}=W_{l} W_{l}^{-1} \bar{x}_{1, f}=\bar{x}_{1, f} .
$$

Now let us consider the subsystem (6b). To solve the problem we define the matrix 99

$$
W_{\mu}=R_{\mu} \widetilde{Q}_{2}^{-1} R_{\mu}^{T} \in \Re^{n_{2} \times n_{2}},
$$


where $R_{\mu}$ is the reachability matrix defined by

$$
R_{\mu}=\left[\begin{array}{llll}
B_{2} & N B_{2} & \cdots & N^{\mu-1} B_{2}
\end{array}\right]
$$

and

$$
\widetilde{Q}_{2}=\operatorname{blockdiag}\left[\begin{array}{lll}
\widetilde{Q}_{2}^{-1} & \cdots & \widetilde{Q}_{2}^{-1}
\end{array}\right] \in \Re^{\mu m \times \mu m} .
$$

If the system (1) is reachable in $q$ steps then the input sequence

$$
u_{\mu}=\left[\begin{array}{c}
u_{\mu-1} \\
u_{\mu-2} \\
\vdots \\
u_{0}
\end{array}\right]=\widetilde{Q}_{2}^{-1} R_{\mu}^{T} W_{\mu}^{-1} \bar{x}_{2, f} \in \Re^{\mu m}
$$

steers the subsystem (6b) from $\bar{x}_{20}=0$ to $\bar{x}_{2, f}$ since

$$
\bar{x}_{2, \mu}=R_{\mu} \hat{u}_{\mu}=R_{\mu} \widetilde{Q}_{2}^{-1} R_{\mu}^{T} W_{\mu}^{-1} \bar{x}_{2, f}=W_{\mu} W_{\mu}^{-1} \bar{x}_{2, f}=\bar{x}_{2, f},
$$

Finally, we define the matrices

$$
\widetilde{Q}=\left[\begin{array}{cc}
\widetilde{Q}_{1} & 0 \\
0 & \widetilde{Q}_{2}
\end{array}\right], R_{q}=\left[\begin{array}{cc}
R_{l} & 0 \\
0 & R_{\mu}
\end{array}\right], W_{q}=\left[\begin{array}{cc}
W_{l} & 0 \\
0 & W_{\mu}
\end{array}\right]
$$

and the input sequence can be computed from

$$
\hat{u}_{q}=\left[\begin{array}{l}
u_{l} \\
u_{\mu}
\end{array}\right]=\widetilde{Q}^{-1} R_{q}^{T} W_{q}^{-1} \bar{x}_{f} \in \mathfrak{R}^{q m} .
$$

The vector

$$
\bar{x}_{f}=\left[\begin{array}{c}
\bar{x}_{1, f} \\
\bar{x}_{2, f}
\end{array}\right], \bar{x}_{1, f} \in \mathfrak{R}^{n_{1}}, \bar{x}_{2, f} \in \mathfrak{R}^{n_{2}}
$$

is related with $x_{f} \in \mathfrak{R}^{n}$ by (4).

Theorem 4 Let the system (1) be reachable in $q$ steps and $\bar{u}_{i} \in \mathfrak{R}^{q m}, i=0,1, \ldots, q-1$ be an input sequence that steers the state of the system (1) from $x_{0}=0$ to $x_{f}=\mathfrak{R}^{n}$. Then the input sequence (23) also steers the state of the system from $x_{0}=0$ to $x_{f}=\mathfrak{R}^{n}$ and minimizes the performance index $(11)$, i.e. $I(\hat{u}) \leqslant I(\bar{u})$. The minimal value of the performance index (11) is given by

$$
I(\hat{u})=x_{f}^{T} W_{q}^{-1} x_{f}
$$

Proof The proof is similar to the proof in $[13,17]$. 


\section{Procedure and example}

The optimal input sequence (23) and the minimal value of the performance index (25) can be computed by the use of the following procedure.

\section{Procedure 1}

Step 1. Knowing $E, A \in \Re^{n \times n}, B \in \mathfrak{R}^{n \times n}$ find matrices $P, Q \in \Re^{n \times n}$ and using (3), (6c) compute $A_{1} \in \Re^{n_{1} \times n_{1}}, B_{1} \in \Re^{n_{1} \times n}, B_{2} \in \mathfrak{R}^{n_{2} \times n}, N \in \mathfrak{R}^{n_{2} \times n_{2}}$.

Step 2. Knowing the matrix $\widetilde{Q}_{1}$ and using (12)-(13) compute the matrices $R_{l}$ and $W_{l}$.

Step 3. Knowing the matrix $\widetilde{Q}_{2}$ and using (17)-(18) compute the matrices $R_{\mu}$ and $W_{\mu}$.

Step 4. Using (4) find the vector $\bar{x}_{f}$ for given $x_{f}$.

Step 5. Using (22) and (23) find the desired input sequence $u_{i} \in \mathfrak{R}^{q m}$, $i=0,1, \ldots, q-1$.

Step 6. Using (25) compute the minimal value of the performance index.

\section{Example 1}

Consider the descriptor discrete-time linear system (1) with matrices

$$
E=\left[\begin{array}{lll}
1 & 0 & 0 \\
0 & 1 & 0 \\
0 & 0 & 0
\end{array}\right], A=\left[\begin{array}{ccc}
0 & 1 & 0 \\
1 & 0 & -1 \\
0 & 0 & 1
\end{array}\right], B=\left[\begin{array}{l}
0 \\
1 \\
1
\end{array}\right] .
$$

The pencil is regular since

$$
\operatorname{det}[E z-A]=-z^{2}+1 \neq 0
$$

In this case

$$
P=\left[\begin{array}{lll}
1 & 0 & 0 \\
0 & 1 & 1 \\
0 & 0 & 1
\end{array}\right], Q=\left[\begin{array}{lll}
1 & 0 & 0 \\
0 & 1 & 0 \\
0 & 0 & 1
\end{array}\right]
$$


and

$$
\begin{gathered}
{\left[\begin{array}{cc}
I_{n_{1}} & 0 \\
0 & N
\end{array}\right]=P E Q=\left[\begin{array}{lll}
1 & 0 & 0 \\
0 & 1 & 0 \\
0 & 0 & 0
\end{array}\right],} \\
{\left[\begin{array}{cc}
A_{1} & 0 \\
0 & I_{n_{2}}
\end{array}\right]=P A Q=\left[\begin{array}{lll}
0 & 1 & 0 \\
1 & 0 & 0 \\
0 & 0 & 1
\end{array}\right],} \\
{\left[\begin{array}{l}
B_{1} \\
B_{2}
\end{array}\right]=P B=\left[\begin{array}{l}
0 \\
2 \\
1
\end{array}\right] .}
\end{gathered}
$$

Therefore, $n_{1}=2$ and $n_{2}=1$. The system (26) is reachable since condition (10) is met. Find the input sequence $u_{i} \in \mathfrak{R}^{m}, i=0,1, \ldots, q-1$ that steers the state of the system from zero state to final state $x_{f}=\left[\begin{array}{lll}1 & 1 & 1\end{array}\right]^{T}$ ( $T$ denotes the transpose) and minimizes the performance index (11) with

$$
\widetilde{Q}=\left[\begin{array}{lll}
2 & 0 & 0 \\
0 & 2 & 0 \\
0 & 0 & 2
\end{array}\right]
$$

From (30) it follows that

$$
\widetilde{Q}_{1}=\left[\begin{array}{ll}
2 & 0 \\
0 & 2
\end{array}\right], \widetilde{Q}_{2}=[2] .
$$

Using the Procedure 1 we obtain the following:

Step 1. Matrices $A_{1} \in \mathfrak{R}^{n_{1} \times n_{1}}, B_{1} \in \mathfrak{R}^{n_{1} \times n}, B_{2} \in \mathfrak{R}^{n_{2} \times n}, N \in \mathfrak{R}^{n_{2} \times n_{2}}$ are given by (29). Step 2. Using (12)-(13), (29), (31) we obtain

$$
R_{l}=\left[\begin{array}{ll}
B_{1} & A_{1} B_{1}
\end{array}\right]=\left[\begin{array}{ll}
0 & 2 \\
2 & 0
\end{array}\right]
$$

and

$$
W_{l}=R_{l} \widetilde{Q}_{1}^{-1} R_{l}^{T}=\left[\begin{array}{ll}
0 & 2 \\
2 & 0
\end{array}\right]\left[\begin{array}{cc}
0.5 & 0 \\
0 & 0.5
\end{array}\right]\left[\begin{array}{ll}
0 & 2 \\
2 & 0
\end{array}\right]=\left[\begin{array}{ll}
2 & 0 \\
0 & 2
\end{array}\right]
$$

Step 3. Using (17)-(18), (29), (31) we obtain

$$
R_{\mu}=\left[B_{2}\right]=[1]
$$


and

$$
W_{\mu}=R_{\mu} \widetilde{Q}_{2}^{-1} R_{\mu}^{T}=1 \cdot 0.5 \cdot 1=0.5 .
$$

Step 4. The matrix $Q=I_{3}$ then $\bar{x}_{f} x_{f}$.

Step 5. Using (22) we get

$$
R_{q}=\left[\begin{array}{cc}
R_{l} & 0 \\
0 & R_{\mu}
\end{array}\right]=\left[\begin{array}{ccc}
0 & 2 & 0 \\
2 & 0 & 0 \\
0 & 0 & 1
\end{array}\right], W_{q}=\left[\begin{array}{cc}
W_{l} & 0 \\
0 & W_{\mu}
\end{array}\right]=\left[\begin{array}{ccc}
2 & 0 & 0 \\
0 & 2 & 0 \\
0 & 0 & 0.5
\end{array}\right] .
$$

From (23) we find the desired input sequence

$$
\begin{aligned}
& \hat{u}_{q}=\widetilde{Q}^{-1} R_{q}^{T} W_{q}^{-1} \bar{x}_{f}= \\
& {\left[\begin{array}{ccc}
0.5 & 0 & 0 \\
0 & 0.5 & 0 \\
0 & 0 & 0.5
\end{array}\right]\left[\begin{array}{lll}
0 & 2 & 0 \\
2 & 0 & 0 \\
0 & 0 & 1
\end{array}\right]\left[\begin{array}{ccc}
0.5 & 0 & 0 \\
0 & 0.5 & 0 \\
0 & 0 & 2
\end{array}\right]\left[\begin{array}{l}
1 \\
1 \\
1
\end{array}\right]=\left[\begin{array}{c}
0.5 \\
0.5 \\
1
\end{array}\right] .}
\end{aligned}
$$

Step 6. Using (25) we compute the minimal value of the performance index

$$
I\left(\hat{u}_{q}\right)=x_{f}^{T} W_{q}^{-1} x_{f}=\left[\begin{array}{lll}
1 & 1 & 1
\end{array}\right]\left[\begin{array}{ccc}
0.5 & 0 & 0 \\
0 & 0.5 & 0 \\
0 & 0 & 2
\end{array}\right]\left[\begin{array}{l}
1 \\
1 \\
1
\end{array}\right]=3 .
$$

\section{Concluding remarks}

Necessary and sufficient conditions for the reachability of the descriptor discretetime linear systems have been given (Theorem 3). The minimum energy control problem for the descriptor discrete-time linear systems by the use of Weierstrass-Kronecker decomposition has been formulated and solved. A procedure for computation of the optimal input and the minimal value of the performance index has been proposed. The effectiveness of the procedure has been demonstrated on the example of descriptor discretetime linear system.

The presented method can be extended to fractional and positive descriptor discretetime linear systems with unbounded and bounded inputs. 


\section{References}

[1] R. BRU, C. COLL and E. SANChEZ: About positively discrete-time singular systems. In Mastorakis M.E. (Ed.), System and Control: Theory and Applications. World Scientific and Engineering Society, Athens, 2000, 44-48.

[2] R. Bru, C. Coll, S. Romero-Vivo and E. SAnchez: Some problems about structural properties of positive descriptor systems. In Benvenuti A., de Santis A. and Farina L. (Eds.), Positive Systems. Lecture Notes in Control and Information Sciences. 294 2003, Springer, Berlin, 233-240.

[3] S.L. Campbell, C.D. Meyer and N.J. Rose: Applications of the Drazin inverse to linear systems of differential equations with singular constant coefficients. SIAM J. on Applied Mathematics, 31(3), (1976), 411-425.

[4] L. DAI: Singular Control Systems. Lecture Notes in Control and Information Sciences. Springer-Verlag, Berlin, 1989.

[5] M. Dodig and M. Stosic: Singular systems state feedbacks problems. Linear Algebra and its Applications, 431(8), (2009), 1267-1292.

[6] G.R. DuAn: Analysis and Design of Descriptor Linear Systems, Springer, New York, 2010.

[7] M.M. FAHMY and J. O'ReILl: Matrix pencil of closed-loop descriptor systems: Infinite-eigenvalues assignment. Int. J. of Control, 49(4), (1989), 1421-1431.

[8] L. FARINA and S. RinAlDi: Positive Linear Systems; Theory and Applications. J. Wiley, New York, 2000.

[9] F.R. Gantmacher: The Theory of Matrices. Chelsea Pub. Comp., London, 1959.

[10] T. KACZOREK: An extension of Klamka's method of minimum energy control to fractional positive discrete-time linear systems with bounded inputs. Bulletin of the Polish Academy of Sciences - Technical Sciences, 62(2), (2014), 227-231.

[11] T. KaCzoreK: Descriptor positive discrete-time and continuous-time nonlinear systems. Proc. of SPIE, 9290 2014, 1-11.

[12] T. KaCZOREK.: Linear Control Systems, vol. 1, Research Studies Press. J. Wiley, New York, 1992.

[13] T. KaCzoreK: Minimum energy control of descriptor positive discrete-time linear systems. COMPEL, 33(3), (2014), 976-988. 
[14] T. KaCzoreK: Minimum energy control of fractional positive continuous-time linear systems. Bulletin of the Polish Academy of Sciences - Technical Sciences, 61(4), (2013), 803-807.

[15] T. KaCzoreK: Minimum energy control of fractional positive discrete-time linear systems with bounded inputs. Archives of Control Sciences, 23(2), (2013), 205-211.

[16] T. KaCzoreK: Minimum energy control of positive electrical circuits. Proc. of 19th Int. Conf. of Methods and Models in Automation and Robotics, 2-5 September, (2014), Miedzyzdroje, Poland.

[17] T. KACZoreK: Positive 1D and 2D Systems. Springer-Verlag, London, 2001.

[18] T. KaCzOREK: Positivity and linearization of a class of nonlinear discrete-time systems by state feedbacks. Logistyka, 6 (2014), 5078-5083.

[19] T. KaCzoreK: Positive descriptor discrete-time linear systems. Problems of Nonlinear Analysis in Engineering Systems, 1(7), (1998), 38-54.

[20] T. KaCzOREK: Positive linear systems consisting of n subsystems with different fractional orders. IEEE Trans. Circuits and Systems, 58(6), (2011), 1203-1210.

[21] T. Kaczorek: Positivity and stability of discrete-time nonlinear systems. Proc. of 2nd IEEE Int. Conf. on Cybernetics CYBCONF, 24-26 June, (2015), Gdynia, Poland.

[22] T. Kaczorek: Positive singular discrete time linear systems. Bulletin of the Polish Academy of Sciences - Technical Sciences, 45(4), (1997), 619-631.

[23] T. KACZOREK: Vectors and Matrices in Automation and Electrotechnics. WNT, Warszawa, 1998, (in Polish).

[24] T. KaczoreK and J. Klamka: Minimum energy control of 2D linear systems with variable coefficients. Int. J. of Control, 44(3), (1986), 645-650.

[25] J. Klamka: Controllability of Dynamical Systems. Kluwer Academic Press, Dordrecht, 1991.

[26] J. Klamka: Minimum energy control of 2D systems in Hilbert spaces. System Sciences, 9(1-2), (1983), 33-42.

[27] J. KLAMKA: Relative controllability and minimum energy control of linear systems with distributed delays in control. IEEE Trans. Automatic Control, 21(4), (1976), 594-595.

[28] V. KUCERA and P. ZaGalaK: Fundamental theorem of state feedback for singular systems. Automatica, 24(5), (1988), 653-658. 
MINIMUM ENERGY CONTROL OF DESCRIPTOR DISCRETE-TIME LINEAR SYSTEMS BY THE USE OF WEIERSTRASS-KRONECKER DECOMPOSITION

[29] P. VAN DoOREN: The computation of Kronecker's canonical form of a singular pencil. Linear Algebra and its Applications, 27 (1979), 103-140.

[30] E. VIRNIK: Stability analysis of positive descriptor systems. Linear Algebra and its Applications, 429(10), (2008), 2640-2659. 